\title{
A Quantum Representation of the Homogeneous 5D Manifold and the Perelman Mappings of 5D onto Non-Homogeneous Lorentz 4D Manifolds
}

\author{
Kai Wai Wong ${ }^{*}$, Peter Chin Wan Fung ${ }^{2}$, Wan Ki Chow ${ }^{3}$ \\ ${ }^{1}$ Department of Physics and Astronomy, University of Kansas, Lawrence, USA \\ ${ }^{2}$ Department of Physics and Centre on Behavioral Health, University of Hong Kong, Hong Kong, China \\ ${ }^{3}$ Department of Building Services Engineering, The Hong Kong Polytechnic University, Hong Kong, China \\ Email: *kww88ng@gmail.com
}

How to cite this paper: Wong, K.W., Fung, P.C.W. and Chow, W.K. (2019) A Quantum Representation of the Homogeneous 5D Manifold and the Perelman Mappings of 5D onto Non-Homogeneous Lorentz 4D Manifolds. Journal of Modern Physics, 10, 557-575.

https://doi.org/10.4236/jmp.2019.105039

Received: March 26, 2019

Accepted: April 23, 2019

Published: April 26, 2019

Copyright $\odot 2019$ by author(s) and Scientific Research Publishing Inc. This work is licensed under the Creative Commons Attribution International License (CC BY 4.0).

http://creativecommons.org/licenses/by/4.0/

\begin{abstract}
The expression of the Maxwell magnetic monopole was employed to correlate the space to space projection that gives rise to the Gell-Mann standard model, and space to time projection which gives the leptons; and how does it correlate to the Perelman mappings from the homogeneous 5D manifold to the Lorentz $4 \mathrm{D}$ manifold, together with correlating the physical consequences caused by the breaking of the Diagonal Long Range Order [DLRO] of the monopoles quantum states affected by the motion of massive particles in the Lorentz $4 \mathrm{D}$ boundary of the $5 \mathrm{D}$ manifold, which leads to gravitons and the gravity field via the General Relativity covariant Riemannian 4D curvatures metric equation.
\end{abstract}

\section{Keywords}

5D Homogeneous Manifold, Perelman Mappings, Magnetic Monopoles, Space Projections and Topological Symmetries, Covariant Riemannian Curvature and Gravity

\section{Introduction}

The homogeneous 5D manifold was presented several years back [1] to explain mainly how the Gell-Mann standard model [2] can be created from the homogeneous symmetry breaking via space dimension reduction projections. The quantitative results derived for the hadrons were subsequently reported [3] [4] [5]. However, there remains mathematical gaps without more investigations into 
the correlations between the quantum picture and the Perelman topological mappings [6] [7] [8]. To start with, we revisit the properties of fields in the homogeneous 5D manifold.

The homogeneous 5D manifold obeys Fermat's Last Theorem [9].

$$
[c t]^{2}=r^{2} \text {. }
$$

Any energy expressed in terms of $2 h v$, would generate a monopole of strength $\pm 2 e c[10]$ [11] outward along " $r$ ", leading to the 5th component Maxwell magnetic monopole, with strength $M=2 e c$, at $r=0$, potential

$$
\pm 2 \frac{\vec{M} \mu}{r} \text {. }
$$

The factor 2 comes from total spin 0 degeneracy of the pair of $e, p, s ;-e,-p,-s$, where $s$ is up or down spin of the massless spinors, and momentum $p$ is along $r$ such that this $\vec{M}$ state is in the DLRO and $\mu$ is the magnetic permeability. The potential has the conventional unit of Joule.

In order to understand properly the process of physical generation of mass and charges from the homogeneous 5D space-time manifold, it is essential to first construct this 5th component of the Maxwell magnetic monopole potential.

The 5D homogeneous space-time manifold, together with the uncertainty principle, results in the presence of 5 vector components potential fields. The first 4 vector potentials $A(0)$ to $A(3)$ are those of the Maxwell electro-magnetic vector potentials, while the 5th component $A(4)$ is explicitly derived recently for the representation of the $5 \mathrm{D}$ as specified by the massless spinor fields expressible in $t \mathrm{U} 3 \mathrm{D} \times 1 \mathrm{D}$ [11]. Topologically, the Maxwell potentials can be represented by a product of two domains: $4 \mathrm{D} \times 1 \mathrm{D}$, such that the monopole potential is orthogonal to the other 4 Maxwell vector potentials, which was originally coined by Maxwell as the magnetic mono-potential [10], and expressed in the recent Physics Essays paper as positive and negative outward spherical radial (generalized) current with a strength of $M=2 e c$, having the unit of ampere-meter. In fact $A(4)$ does not have the same dimension as $A(0)$ to $A(3)$, although the potential energy $M A(4)$ must be the same in unit as $e A(0)$. This magnetic monopole can be viewed as a combination of numerous $M$ vectors propagating outward along all radial unit vectors at the same rate $c$, while the $5 \mathrm{D}$ manifold expands. Therefore, it carries any momentum $\frac{h \vec{v}^{\prime}}{c}$ where $\vec{v}^{\prime}$ has no relationship to the frequency $v$ of the pair of charged massless spinors, with its values only dictated by the Fermat's amplitude $r$, like the photon that may be emitted from the Coulomb potentials and also obeys Gauss Theorem, with the $\vec{M}$ vector replacing the electric field vector $\overrightarrow{\boldsymbol{E}}$. These monopole strengths $M$ arise from the outward radial currents due to opposite momentum pairs of $e,-e$ massless spinors created from pure energy, where the massless charges $e,-e$ represent the coupling constants between the vector fields and the spinor fields solutions of the equation with the homogeneous 5D energy-momentum quadratic operator acting on the wave function of the system. Such that these vector and spinor fields obey gauge invariance. Hence, $\vec{M}$ and $-\vec{M}$ are quantum Bosons composed of 3D 
radial outward massless $e, p, s ;-e,-p,-s$ current states naturally created from energy at the center of the $5 \mathrm{D}$ manifold. As long as the $5 \mathrm{D}$ manifold is not totally enclosed by matter, the vectors $\vec{M}$ and $-\vec{M}$ and hence the magnetic monopole will exist. Mathematically, these vectors $\vec{M}$ and $-\vec{M}$ are massless bosonic states with strength $2 e c$ that represents the magnetic monopole. In fact, the constituent vectors representing the monopole expand spherically outward like photons in the 4D homogeneous EM space-time manifold, except they carry only energy with arbitrary momentum, only fixed by the b.c. of the Fermat's amplitude $r$. Hence they must be in the Diagonal Long Range Order (DLRO) Bose-Einstein (BE) condensate state. Because of this B.E. ground state, we could also see certain similarities between a $4 \mathrm{D}$ space-time manifold with the $M$ and $-M$ Bose condensate states and the Higg's theory Bose-Einstein condensed ground state/vacuum filled with Higgs fields [12]. Of course the premises of these two theories are quite different.

Should we be able to add a net linear momentum, from the motion of a massive nuclear in the $r=0$ 5D frame, with charge 0 or $q$ to the monopole inducing current magnitude $M$ and $-M$, The currents generating magnetic field will be replaced by $q \vec{v}+\vec{M} N(+)$ and $q \vec{v}-\vec{M} N(-)$, where $\vec{v}$ is the velocity of charge $q$. Where $N(+)$ and $N(-)$ are the energy integrated weight factors, then these two opposite magnetic monopoles will give raise to an attractive quantum well in the $5 \mathrm{D}$ manifold, as it changes the monopole strength to $M^{\prime}=q \vec{v}+\vec{M} N(+)$ and $-\vec{M}$ " $=q \vec{v}-\vec{M} N(-)$ Where $\vec{v}$ need not be along " $r$ ". Unlike $M$, which is a Boson state, the nuclear charge $+q$ can be either a Fermion or a Boson. Hence the Fermionic and Bosonic statistics of $\vec{M}^{\prime}, \vec{M}^{\prime \prime}$ is broken and gives us the attracting effect on the massive " $q$ " by a $V(5)$ monopole-monopole potential well:

$$
V(5)=-\frac{M^{\prime} M^{\prime \prime} \mu}{r} \cong-\frac{[2 e c]^{2} N(+) N(-) \mu}{r}
$$

with " $r$ " fixed by $c t$, irrespective of whether $t$ is fixed or not.

The term $(q v)^{2}$ can usually be neglected, as it is normally extremely small for the nucleus of $q$ charge as $N(+)$ and $N(-)$ are canonical ensemble generated, temperature-dependent, large thermal quantities. As such $V(5)$ is valid only for a macro thermal system, not the initial space dimension projections that created the basic leptons and hadrons, similar to the Perelman-entropy mapping. However when $q \vec{v}$ exist, the boundary conditions of $\vec{M}^{\prime}$ and $-\vec{M}^{\prime \prime}$ are altered, and $\vec{M}^{\prime}$ as well as $-\vec{M}^{\prime \prime}$ are no longer Bosonic and hence in the Bose condensate state.

It should be pointed out that due to the Pauli exclusion on the e-trino, anti-e-trino, the repulsive magnitude $M^{2}$ is given by the products of $(e, p) \times(-e,-p) \times\left(e, p^{\prime}\right) \times\left(-e,-p^{\prime}\right)$. Where $(e, p)$ and $(-e,-p)$ represents the $e$-trino and anti-e-trino spinors with $p$ and $-p$ momentum, that is only radially outward, so sign cannot be changed. Note that e-trino state commutes with anti-e-trino states, while anti-commutes with another $e$-trino state. Thus 
$M^{2}$ can be expressed as

$$
\frac{1}{4}\left\{(e, p) \times\left(e, p^{\prime}\right)-\left(e, p^{\prime}\right) \times(e, p)\right\} \times\left\{(-e,-p) \times\left(-e,-p^{\prime}\right)-\left(-e,-p^{\prime}\right) \times(-e,-p)\right\} .
$$

The $e$-trino and anti-e-trino pair states are Boson. But due to its antisymmetry form for arbitrary $p$ and $p^{\prime}$, its thermal average has 0 net weight.

On the other hand the attractive monopole-monopole magnitude comes from the product between $M$ and $-M$, where the $-M$ vector is obtained by interchanging $e$ with $-e$ and vice versa in the definition of $M$ giving by the radial current $2 e c$. This change will no longer lead to the antisymmetry of the e-trino pairs and the thermal weight factor is not 0 .

Since the 5D manifold irrespective of " $t$ " is given by Fermat's sum, and with charge conservation, therefore irrespective of the $4 \mathrm{D}$ space as long as there is a finite size of the opening for the 1D subspace that allows for $r$ along that 1D expansion, $N(+)=N(-)=N$. In other words, the monopole potential well becomes space inhomogeneous. In fact $N$ would be proportional to the ratio of the solid angle " $S$ " of the opening as compared to the spherical solid angle $4 \pi$, and is a linear function of the temperature, as $M$ (having the unit of ampere-meter) represents a boundary-condition-dependent Bosonic state, with degenerate energy $E . V(5)$ is thus modified by $s=S / 4 \pi$. And for first order approximation we should modify $N(+) N(-)$ by $N(+) N(-) s$. And we can get from the Bose distribution the thermal number of such $M$ states

$$
N=a \text {. }
$$

where $a$ is derived from integrating the Bose distribution over $\frac{d E}{k T}$ from $\frac{h}{t}\left(\frac{n}{k T}\right)=C$, where $n$ is an arbitrary number, such that at $t=t_{o}$, the time of the Big Bang, we have $\frac{n h}{t_{o}}=h v(o)$ which must exceed the rest energy of the quark rest mass energy $m(Q) c^{2}$, such that at least it is enough to create both electrons and protons and neutrons in the Lorentz boundary domain, while maintaining net charge neutrality. The higher the temperature $T$, the smaller will be $C$ when $h v(o)$ is fixed, leading to a larger $N$ number and thereby more massive particles created in the Lorentz boundary domain which implies in the nucleus more fusion, and in the stars and planets more mass, even implying a larger galaxy. Hence the quantity $C$ corresponds to the chemical potential of the grand canonical ensemble. Should $t \rightarrow 0, C \rightarrow \infty, a \rightarrow 0$, implying without extra input of starting creation of the monopole potential fields at a finite initial $t(o)>0$ value, the homogeneous 5D manifold cannot have any $M$ as well as Maxwell EM potentials, irrespective of the uncertainty principle, because there were no sources in the 5D manifold. However with $C>0$ and finite

$$
N=\frac{4 \pi k T}{h} \sum_{j=1}^{\infty} \frac{e^{-j C}}{j}<\infty
$$

where $j$ is a positive integer. 
This sum " $a$ " is finite, and proportional to $T$, the absolute temperature, implying also that the quantum well cannot be completely cancelled by a finite number of + or - massive charges created. On the other hand, if the monopoles $M$ and $-M$ are replaced by $M^{\prime}$ and $-M^{\prime \prime}$ the Bose statistic, and the Bose condensation is broken, similar to a physical feature employed by Higg's theory. With the quantum well potential completely changed by equal number of relativistic massive charges of opposite signs in the $4 \mathrm{D}$ Lorentz boundary domain.

The mass associated with the $+q=n e$ charge is not necessarily equal to that of the sum of $n-e$ massive spinors. Both masses from the $5 \mathrm{D}$ field theory are the result from space projection into $4 \mathrm{D}$ Lorentz manifolds via the space to time projection $P_{0}$, and the pure space dimension reduction projection from $4 \mathrm{D}$ to $3 \mathrm{D}$ through $P_{1}$ enacted during the creation of the 5D universe, at $t(o)>0$ the instant of the Big Bang, when the monopoles must be created. It was assumed in the $5 \mathrm{D}$ book such projections will not happen again in later time [1]. The modification of the monopole $\mathrm{M}$ by the massive charge term $q \vec{v}$, changes the resultant monopole statistics and thus no longer can be in Bose-Einstein condensation, unless we transform to the stationary frame of the massive $q$. It is this frame transformation, that recovers the quantum well, thus brings on the total enclosure of the $5 \mathrm{D}$ by matter via nuclear fusion into a heavy nucleus and eventually with a $5 \mathrm{D}$ time frozen center void.

The difference between $\vec{M}^{\prime}$ and $-\vec{M}^{\prime \prime}$ comes from the symmetric pairs of $e$ and $-e$ within $\vec{M}$ and $-\vec{M}$ with different energy values partly converted into $q=+e n$ massive nucleus of mass $m(+)$ or $n,-e$ massive electron spinors, with electron mass $m(e)$ via $P_{0}$ the 4 th space component projection onto the time axis, mapping $5 \mathrm{D}$ into Lorentz $4 \mathrm{D} \times \mathrm{SU}(2)$, where $\mathrm{SU}(n)$ represents the semi-simple compact Lie Groups of $n^{\prime}$ dimensions, and from $+e$ massive spinors [the proton] via $P_{1}$ the 4 th space component onto the remaining 3 space components, making up the protons and neutrons of the nucleus, such that the proton is defined by the gauge confined $u, u, d$ quarks, and the neutron as $u, d, d$ quarks; where $u$ is the $\frac{2 e}{3}$ up quark, and $d$ is the $-\frac{e}{3}$ down quark. Or breaking $5 \mathrm{D}$ into Lorentz $4 \mathrm{D} \times \mathrm{SU}(3)$. Hence $m(e) n \ll m(+)$ where $m(e)$ is the lowest energy state, the electron rest mass $m(e)$ as the nucleus creation takes far more energy than the $n$ electrons creation from the energy of the homogeneous 5D Fermat energy-momentum manifold, breaking the quantum homomorphic $\vec{M}$ and $-\vec{M}$ distribution and the Bose-Einstein condensation over the $4 \mathrm{D}$ homogeneous space, as given by the third phase angle of 0 to $4 \pi$ in the Fermat's representation. Such a breaking of the homogeneity of the $5 \mathrm{D}$ manifold must occur first before the deformation of the $5 \mathrm{D}$ space-time into separated $5 \mathrm{D}$ manifolds with separated 4D Lorentz manifolds in the topology of 3D space of doughnut shapes as given by the Perelman-Ricci flow mapping. In fact it is important mathematically to realize the change of $\vec{M}$ and $-\vec{M}$ along " $r$ " of the 3D homogenous space to $\vec{M} N+q \vec{v}$ and $-\vec{M} N+q \vec{v}$, where the massive $q$ 
charge velocity $\vec{v}$ is an arbitrary $3 \mathrm{D}$ vector. This implies the resulting monopole current is no longer necessarily along " $\overrightarrow{ }$ " and will no longer obey Gauss Theorem. It is this feature that implies that the monopole vector potential must be expressed in a covariant representation due to the motion of the massive charge $q$, similar to the treatment of the Coulomb potential of a charge $q$. It is this correlation that leads us to the Riemannian curvature for the Lorentz space-time due to the dynamic distribution of masses within, even when these masses can be considered as charge-less on the classical scale, and as the monopole quantum well vanishes when $s$ becomes 0 , or by the change in frame to $\mathrm{V}$, when

$$
s[M N]^{2}=[q v]^{2}=[n e v]^{2}
$$

representing the charge neutral mass case when the simultaneous condition on both the positive $q$ and negative $-n e$ massive charges having the same Fermat's amplitude value $r$, which is the quantum well " $r$ ". Since $N$ is linear in $T$, hence the right hand side of (1.6) which includes $v^{2}$ must also varies as $s T^{2}$. When that happens, the charge neutral moving mass in the Lorentz $4 \mathrm{D}$ domain observes no monopole well effect, and the nuclear fusion ceased, and the remaining binding is replaced by the forming of atoms by Coulomb attraction between the nucleus and its orbiting electrons. But most important it gives us a relationship between how $s \rightarrow 0$ due to $v^{2}$ of the nucleons in the nucleus mass shell, forming the Poincare sphere, as evolved through the Perelman-entropy mapping. Using the expression for $N$ as an infinite series sum as given by (1.5) the relationship between $s$ and $N$, can be expressed as a double sum series equation

$$
\sum_{j=1}^{\infty} \sum_{j^{\prime}=1}^{\infty} \frac{s\left(j, j^{\prime}\right)}{j j^{\prime}} e^{-\left(j+j^{\prime}\right) C}=K,
$$

where $K$ is a constant.

As $\vec{M}$ and $-\vec{M}$ are by uncertainty principle required to be along the same vector $\vec{r}$ of the 5D manifold so that $V(5)=M A(4)$ is also a solution of the $5 \mathrm{D}$ metric operator, hence $s\left(j, j^{\prime}\right)$ must be confined by a gauge restriction of $\frac{h c}{M}=\frac{h}{2 e}$, which defines a gauge loop and thereby the Boson field $M$ can be decoupled by a gauge transformation. This loop integration requires defining a $Z$ vector direction, and coupled to the conversion of $\vec{M}$ and $-\vec{M}$ into 2 closed current loops of $J$ or $-J$, where the magnitude $J$ is still 2ec, but is no longer of DLRO, and made of the pair $e, p x r^{\prime}, s$; and $-e,-p x r^{\prime}, s^{\prime}$, and separated along $Z$, in a $2 \mathrm{D} \times 1 \mathrm{D}$ space representation, where the spins $s, s^{\prime}$ are not necessarily opposite, and the gauge loop radius that decouples $M$ from A(4) for $r^{\prime}<r$, the Fermat's amplitude. Such a single closed $J$ loop also leads to $e,-e$ annihilation, unless $J$ is split into 2 parallel but separated ec closed loops, which would generate a magnetic dipole field, and obeys Chern-Simons gauge, leading thus to a Perelman-Ricci Flow mapping.

Hence $s\left(j, j^{\prime}\right)$ has a solution depending on 


$$
e^{-\left(j+j^{\prime}\right) C}
$$

where the chemical potential $C$ is a positive number. In fact $C$ must be related to the $q$ and $\frac{n e}{k T}$ the open $5 \mathrm{D}$ core of $2 \mathrm{D}$ radius " $r$ ". This form of $s>0$ corresponds to the Bose condensation of $N$ being broken by the presence of $q \vec{v}$. Therefore the higher the $3 \mathrm{D} \times 1 \mathrm{D}$ core temperature the more nuclear and electron matter will have to be created on the final closed shell.

Its relationship to $N$ is the physics that basically gives us the Perelman-entropy mapping from the Perelman-Ricci Flow open doughnut Lorentz manifold into the closed Poincare sphere, when the isolated monopole no longer exist. This triple relation between $q=n e, \frac{v}{c}$ and $N$, a function of increasing temperature will result in a phase diagram for elements formed at $T$. Hence the complete periodic table is reached in cool systems. Thereby, the pure gravity field observed by the observer must be derived from the covariant Riemannian $4 \mathrm{D}$ space-time, chosen fixed by the rest frame of the observer fixed on the moving masses formed by elements that gives us the Newtonian gravitation potential of a stationary mass as derived from the curvature metric equation according to General Relativity.

As we mentioned without defining a $t(o)>0$ for the Big Bang, the 5D homogeneous manifold contains no fields of any kind. Hence in the process of creation of the monopoles at $t(o)$, there must be the total energy conservation given by

$$
\langle E\rangle+V(5)=0
$$

The quantity $\langle E\rangle$ represents the total ensemble energy of creating the monopoles $\vec{M}$ and $-\vec{M}$. It is easy to see that both terms in (1.9) are proportion to $T^{2}$. Hence the temperature of the manifold cannot be determined, by $C$ and $s$.

\section{The Force Equation in the Non-Homomorphic 5D Manifold within the Magnetic Monopole Attractive Quantum Well for a Massive $+q$ Charge}

The presents of $V(5)$ creates an energy sink in the $3 \mathrm{D} \times 1 \mathrm{D}$ space of the $5 \mathrm{D}$ manifold, hence any matter in the 4D Lorentz boundary domain would be attracted towards the center of the $5 \mathrm{D}$ manifold, the $2 \mathrm{D} \quad r^{\prime}=0$, similar to the presence of a black hole in 4D General Relativity theory. But in 5D theory, matter cannot exist inside the 1D monopole subspaces. This requirement can be achieved if the matter in the Lorentz $4 \mathrm{D}$ boundary domain has an angular momentum $L$, such that as its distance $R>r^{\prime}$ to the void deceases towards $r^{\prime}$, of the 1D $M$ domain, its energy increases so that its momentum $\rho$ increases, leading to a centrifugal outward force sufficient to counter the monopole quantum well attractive strength, by converting part of the energy carried by the $\vec{M},-\vec{M}$ states along $r$ into coherent rotating $e,-e$ massless counter $L$ state around $r^{\prime}$ to the revolving mass $L$, via the reducing of the covering solid angle ratio $s$, eventually $s$ 
becomes 0 , enacting the Perelman-entropy mapping in the maintaining of balance of net $L=0$ as the monopole $M$ 's energy distribution in the homogeneous $5 \mathrm{D}$ is reduced to 0 . This feature was discussed earlier by us [13] as the origin for Astro objects having self rotation.

As discussed in the introduction a single isolated $m(+)$ massive nucleus with charge " $+q$ " within the non-homomorphic $5 \mathrm{D}$ manifold is subjected to the quantum approximate well given by $V(5)$, thus at a given time $t$, it must be governed by the force balanced equation, equivalent to the introducing of the Lagrangian for the Ricci-Flow mapping given by Perelmann [7]. In fact there are Two separate Ricci Flow directions: One from the split of the 2 in phase rotation states of the $e$-trino and anti-e-trino from $M+(-M)$ 'which carries energy outward along $r^{\prime}$ [note all $M$ are outward radial vectors] into either $L z$ or $-L Z$ angular momentum as $\mathrm{BE}$ of the monopoles is broken; the other is from breaking a $M=2$ ec into 2 separated and perpendicular to $z^{\prime}$ parallel closed loops of in phase current ec each due to gauge that changes $M$ to give a dipolar magnetic field $H z^{\prime}$, or $-H z^{\prime}$ where $z^{\prime}$ is not aligned to $z$ of $L z^{\prime \prime}$ in the 5D domain. The non-selected opposite states for angular momentum and magnetic field values are then replaced by the responses from the masses in the Lorentz boundary domain. Thus giving the force balancing equation:

$$
\frac{q H v}{c}-\frac{m(+) v^{2}}{r^{\prime}}+\frac{\mathrm{d} V(5)}{\mathrm{d} r^{\prime}}=0
$$

The first term is the Lorentz force, due to a $H$ field generated by all the moving charges within the $4 \mathrm{D}$ Lorentz manifold on $q$, and including the dipolar field generated by the non DLRO closed $J$ or $-J$ loops. The dipole $H$ field obtained from $J$ or $-J$ is then given by the loop currents thermal averaged over product of two Fermi distributions of $e, p x r^{\prime}$ and $-e,-p x r^{\prime}$; [see Fung and Wong [14] on the $\mathrm{Hz}$ field of stars, JMP for details] the second term is the $+q$ nuclei with mass $m(+)$ 's centrifugal force, arising from the outward spiral of $m(+)$ as its $L z^{\prime}$ changes caused by a $-L z^{\prime}$ of pairs of $e$-trino and anti-e-trino in phase rotation within the 5D core; note $z^{\prime}$ cannot be aligned with $\mathrm{Hz}$ as it will lead to the $e$-trino, anti-e-trino annihilation, while the last term comes from the magnetic monopole quantum well, that the charge current " $q v$ " was created via the dimension reduction projection. Thus the " $+q$ " charge sign nuclei Fermat's amplitude " $r$ " " is given approximately according to (2.1) by

$$
r^{\prime}=\frac{\left(m(+) c^{2}\right)\left(\frac{v}{c}\right)}{2 q H}\left\{1+\sqrt{\left[1-\frac{4 M^{2} \mu s N^{2} q H}{\left(m(+) c^{2}\right)^{2}\left(\frac{v}{c}\right)^{3}}\right]} .\right.
$$

There are 3 variables, namely $q v, r$ and $H$. Hence there is no unique solution to " $r$ " ".

But since $r^{\prime}$ must be real and positive, it means 


$$
m(+) c^{2}\left(\frac{v}{c}\right)^{1.5}>2 M N \sqrt{s \mu q H}
$$

As $q=n e$ and $m(+)>m(p) n$ where $m(p)$ is the proton rest mass, due to neutrons within the nucleus. Thus we have

$$
H<\frac{\left(m(+) c^{2}\right)^{2}(v / c)^{3}}{4 \mu q M^{2} s N^{2}}
$$

As $\frac{v}{c}$ according to special relativity is bounded by 1 , the nucleus mass $m(+)$ and the nucleus $r$ then dictates the upper bound value of the magnetic field $H$.

Thus since the nucleus $r^{\prime}>0$, and small, it has an upper bound value $H=\frac{m(+) c^{2}[v / c]}{q N}$. As $N \rightarrow \infty$ as $C \rightarrow 0$ and $M$ is in the Bose condensate state, $H=0$. Even not in the condensed state $H$ value is relatively low because of large but not infinite $N$ when the Bose condensation is broken making nuclear fusion from single proton to nucleus with $n>1$ possible. While there is in general no lower bound on $H$ limitation which is also valid in stars and planets as $r$ increases to astronomical value.

For the electron force equation solution we replace with $-e(-v)$ replacing $q v$ and $r^{\prime}$ changes from (2.2) to

$$
r^{\prime}=\frac{\left(m(e) c^{2}\right)\left(\frac{v}{c}\right)}{2 e H}\left\{1+\sqrt{\left[1-\frac{4 M^{2} \mu s N^{2} e H}{\left(m(e) c^{2}\right)^{2}\left(\frac{v}{c}\right)^{3}}\right]}\right\} .
$$

It is obvious " $r$ " for electron is greater than " $r$ " " for the nucleus with $+q$ charges, even if $\frac{v}{c}$ is the same. Condition on $H$ with $N$ given by (2.4) is modified to

$$
H<\frac{\left(m(e) c^{2}\right)^{2}(v / c)^{3}}{4 e \mu s M^{2} N^{2}}
$$

Since $\frac{v}{c}<1, H$ must be inversely proportional to $N^{2}$. But for the nucleus charge $q$, it is inversely proportional to $n N^{2}$. Thereby the dipolar magnetic $H$ $1 \mathrm{D}$ field has to be weak in heavy nuclei. Furthermore, since $H$ is the same for both, it follows that $m(+)$ must be proportional to $n m(e)$. It should be noted that the $r^{\prime}$ solution for the electron is outside the nucleus " $r$ " " by orders of magnitude, which further reduces the mass ratio $\frac{m(e)}{m(+)}$. This extra ratio comes from the SU(3) Lie Group generators. Therefore for general " $r$ " solutions exceeding that for the $m(e)$ solution given by (2.6) as $N$ and $n$ become very large, would result in the $4 \mathrm{D}$ Lorentz domain with charge neutral matter and then be- 
comes the pure classical topological Perelman mappings derived from the 5D homogeneous manifold. In conclusion, the nucleons, proton and neutron masses are mainly due to quantum gauge confinement of the repulsive gluon potential generated by the intermediate quark currents [1]. While the binding of the nucleons to form heavy nucleus arises from the attractive mono-pole quantum well until the matter shell closes to complete a Poincare sphere, as s becomes 0 , the quantum well disappears.

Astronomical matters, such as galaxies, stars and planets the above force balanced equations is not strictly applicable not because $s$ becomes 0 , but rather that we are in a classical gravity domain, where some physicists believe in the graviton theory, where all matters are formed by charge neutral atoms, as the time frozen void $3 \mathrm{D} \times 1 \mathrm{D}$ way exceeds the dimension of atoms, and with $N$ being an integer, gravity is quantized, in a way similar to photons. Where the Lorentz force and as the monopole potentials no longer apply for the charge neutral matter. None the less, we believe it is the replacement for the monopole attractive quantum well by the Newtonian gravitation potential in the rest frame of the neutral mass $m(+)+m(-)$, with the curving of the Maxwell 4 vector potentials acting on a combined massive large number of charges $q$, and -ne forming atoms and molecules in a pure $4 \mathrm{D}$ Lorentz domain as explained in section 1, as the open surface area $S$ of the Gauss surface shrinks due to the closing by the mass shell around the $3 \mathrm{D} \times 1 \mathrm{D}$ space void it must be considered multiplied together with the infinite $N^{2}$ magnitude of the monopole well, [see eq. 1.5] due to the lower limit in its energy spectrum when in the specific rest mass $4 \mathrm{D}$ Lorentz frame such that the monopole quantum well strength in the mathematical limit $s \rightarrow 0$, as $N \rightarrow \infty$ when $C \rightarrow 0$, such that the quantum well $s N^{2} M^{2}$ strength is replaced by the attractive Newtonian gravity potential with strength $G M(0)$ where $G$ is the Newtonian constant and $M(0)$ the mass of the Poincare sphere. Hence with relative moving masses, as given geometrically as Poincare spheres, the gravity potential field from multiple masses must be derived from the curvature arising from the covariant mass frames creating the warping of the $4 \mathrm{D}$ Lorentz-Riemannian covariant space-time caused by the dynamic distribution of all these masses in relative motions within the $5 \mathrm{D}$ universe, a result discussed in Einstein's General Relativity. Such a non-linear gravity potential equation without the masses having $3 \mathrm{D} \times 1 \mathrm{D}$ voids must then possess a singularity independent to the mass corresponding to the $N$ divergence. In fact it can be easily seen physically from the exact $q \vec{v}+N \vec{M}$ and $q \vec{v}-N \vec{M}$ moving charge currents given in (1.3) as $q \vec{v}$, which is on the Lorentz boundary domain becomes comparable to $N M$ in magnitude, that the approximated $V(5)$ is no longer valid, and the shrinking of the open Gauss surface because of the finite sizes of the matter composed of atoms such that the Perelman entropy mapping takes over and closes the mass into a Poincare sphere, the monopole strength will decrease to zero, breaking the Bose-Einstein condensate state. In another word, $N$ is a decreasing function of $s$ and the final Poincare mass sphere should 
enclose a time frozen $3 \mathrm{D} \times 1 \mathrm{D}$ void, such as that suggested by Wheeler's worm hole [15]. As s shrink to 0, the expanding $M$ along $r=z$ is cut-off. Due to expanding energy continuity, $M$ must be converted into a pair of 180 degree off-phase photons, which carry no $E, H$ amplitudes as it propagates along $z$. Thus such a beam can be detectable from newly formed stars, and could be interpreted as a graviton beam [16]. In fact this topological mapping result not only removes the Schwarzschild singularity of gravity, but it actually removes the concept of 'black holes' in the 5D universe. The idea of black holes in the universe had gained a lot of fantasy among physicists [17]. Recently there is a report of a so called photo sighting of a black hole, if correctly verified which would have to be a V(5) according to the 5D theory. Within an enclosed Poincare sphere, the new quantum perpetual states within the $3 \mathrm{D} \times 1 \mathrm{D}$ void are no longer in $M$ states, rather they are massless spinor pairs that give angular momentum, thus creates a self rotation of the mass shell, and the closed $J$ or $-J$ parallel loops breaking parity that gives a finite dipolar $\mathrm{Hz}$ field as $J$ is split into 2 parallel ec loop currents along $z^{\prime}$ values according to CP and as we had discussed in previous papers ([14], Fung and Wong, JMP). The key to finding these states in thermal equilibrium is the energy cut-off value, that must be put into the Bose distribution of the $M$ and $-M$ states as s decreases which in turn will reduce $s N^{2} M^{2}$ to a finite number proportion to $G M(0)$ thus producing in the mass $M(0)$ rest frame the Newtonian gravitational potential.

\section{The Break of the Homogeneity of the 4D Space, into a Cylindrical Representation Plus the Bending of $M^{\prime}$ and $-M^{\prime \prime}$ Magnetic Monopole States in the Entangled 4th Space Variable with $z$ and $r$ of the 3D Cylinder Variables}

To mathematically understand how the eigen solutions of revolving charge within a dimension reduced space can be equivalent to the revolving charge's mass will give us further understanding on the importance of the monopole quantum well effect on the $4 \mathrm{D}$ covariant Lorentz space time Riemannian curvature.

It was proposed by Perelman that the Ricci Flow topological mapping of the $5 \mathrm{D}$ homogeneous space-time would result in a non-homogeneous $4 \mathrm{D}$ Lorentz manifold, with the $3 \mathrm{D}$ space in the geometrical shape of a doughnut, with a center $5 \mathrm{D}$ open core, such that the 4 th space coordinate 1 'D is the space coordinate through the vector addition from open $z$ axis through the doughnut core center and the closed changing $r$ as a function of $z$, of the doughnut tube variable around the core. It is such a $4 \mathrm{D}$ Lorentz manifold that properly represents the $4 \mathrm{D}$ space-time of a galaxy, where all the stars, planets and meteors are contained. In fact such a mapping can be quantized during the absolute beginning of the $5 \mathrm{D}$ universe matrix, by employing the $P_{0}$ and $P_{1}$ projections from this 4 th 1 'D entangled space representation dimension onto the $t$ and $2 \mathrm{D}$ coordinates via $P_{0}$ and $P_{1}$ mapping. By using a $2 \mathrm{D} \times 1 \mathrm{D} \times 1$ 'D space symmetry representation, the $3 \mathrm{D}$ 
Lorentz gauge invariance must now be replaced by the $2 \mathrm{D}$ Chern-Simons gauge [18] [19]. While the net outward $\vec{M}^{\prime}$ and $-\vec{M}^{\prime \prime}$ can be viewed physically like currents, which included the nuclear and electron currents on Lorentz boundary due to $q v=n e v$ and nev from the charge cancelling electrons forces causes the $\vec{M}^{\prime}$ and $-\vec{M}^{\prime \prime}$ bending along the curving $1^{\prime} \mathrm{D}$ through the $3 \mathrm{D}$ doughnut open center.

Without considering $\vec{M}^{\prime}$ and $-\vec{M}^{\prime \prime}$ on the curving portion, it is interesting to investigate the Hamiltonian for the electron eigenstates just around the core at $z=0$. This Hamiltonian is in $2 \mathrm{D}, \vec{M}^{\prime}$ and $-\vec{M}^{\prime \prime}$ are reduced to only $\vec{M}$ and $-\vec{M}$ along up and down $z$ and making the monopole quantum well $V(5)$ resembles that of the relativistic $2 \mathrm{D}$ hydrogen, with Coulomb like potential $2 \mathrm{D}$ monopole-monopole attractive potential $-4 \frac{e^{2} c^{2} \mu}{r}=-4 \frac{e^{2}}{\varepsilon r}$ due to $\varepsilon \mu=\frac{1}{c^{2}}$. Although the monopole-monopole quantum well is expression wise equivalent to the Coulomb attractive potential of $-4 e^{2}$, topologically it is totally different. Nonetheless the eigen solution of a revolving massive electron would satisfy the Chern-Simons gauge. In the $2 \mathrm{D}$ Coulomb potential the charges are massive and the positive charge $+e$ is the proton at the origin $r=0$, while the much lighter electron is in a relativistic ground state orbit, with the binding energy precisely equal to the electron reduced mass energy. In the case of the $2 \mathrm{D}$ quantum well produced by $M$ and $-M$ along $z$, the massive electron ground state binding energy must then be precisely 4 times the electron rest mass [19]. Therefore, we can interpret it as the $2 \mathrm{D} \times 1 \mathrm{D}$ quantum well containing an orbiting electron with energy equal to $4 m(e) c^{2}$ on the $2 \mathrm{D}$ Lorentz boundary, or in another word the parity broken mono pole well can be cancelled by an energy cut-off to the Bose distribution equal to 4 electrons mass. This same potential well binding if applied to that of a revolving nearly 2000 times heavier proton say would be in a 2D non-relativistic orbital state similar to that given by nuclear shell model. Actually as according to (1.3), there are 4 choices for the $5 \mathrm{D}$ conversion into $4 \mathrm{D}$ $\times 1 \mathrm{D}$ mapping, thus the $N$ value for the $u, u, d$ quarks that makeup the proton should be weighted by the 3 axis $P_{1}$ choices $4 \times 4 \times 4$, a result that also generate the gluon field via gauge confinement that gives the major portion of the proton mass. Hence the monopole quantum well strength should be weighted by 64 for the quarks. Note that all $M$ monopole states are charge neutral. Therefore if a $-e$ massless spinor is projected onto $t$, and becomes an electron, then one $+e$ must also be projected by $P_{1}$ to form a proton. Hence because of the $1 \mathrm{D}$ having a 4 fold choice, in terms of the quark rest mass derived from $m(e)$, must be $4 \times 4 \times 4$ $m(e)$, which is roughly $32 \mathrm{MeV}$. This value for the quark rest mass agrees well with data fitting for the mesons and baryons to within error due to the relativistic modification on the quark constituent masses within each hadron [3] [4] [5]. The relativistic lowest ground state from such a 2D potential irrespective of the monopole or the equivalent Coulomb strength under Chern-Simons gauge gives the binding exactly equal to that of the electron rest mass without change in the electron statistic.

Hence here the 2D relativistic ground state of the monopole quantum well is 
$-4 m(e) c^{2}$. Thereby the total $2 \mathrm{D}$ hydrogen like quantum well after creating the electrons has no more energy [19], The mathematical derivation given in those papers are valid also for the mono-pole quantum well, illustrating the gauge effect on the $P_{0}$ projection of creating electrons in the presence of Perelman-Ricci Flow mapping. In short, the electron mass $m(e)$ is created by the $2 \mathrm{D}$ monopole-monopole attractive quantum well under Chern-Simons gauge transformation via the Perelman-Ricci flow mapping same as the $P_{0}$ projection from the 4 th space component onto time, resulting in an electron placed within a $4 \mathrm{D}$ Lorentz manifold. This interesting approximate result implies that the $\vec{M},-\vec{M}$ 1'D like currents would be continuously ejected from the doughnut 5D core of a galaxy, according to the 5D homogeneous energy-momentum metric, at $t=0, E$ and $p$ are infinite, hence despite the creation of numerous galaxies from the Big Bang, the galactic center of the galaxy will still have infinite amount of energy to create $\vec{M}$ and $-\vec{M}$ magnetic pole currents radiating outward along the $1^{\prime} \mathrm{D}$, although these $\vec{M}$ and $-\vec{M}$ energetic bosons will not be projected to form new $\mathrm{SU}(2) \times L$ and $\mathrm{SU}(3) \times L$ manifolds they can excite the leptons and nucleons causing ionization as well as nuclear fission, and any star system that spirally revolves outward on the Lorentz doughnut surface, would periodically receive these $\vec{M},-\vec{M}$ high energy like monopole beams. Since, $\vec{M}$ and $-\vec{M}$ with high energies above the electron and quark mass must preserve the equal numbers of electrons and protons, causing atomic ionization and nuclear fission, which upon thermal cooling afterward can lead to new nuclear fusion and the changes in the element composition in its matter shell, it means a planet, like earth, which star the sun revolves on the 4D Lorentz doughnut manifold would necessarily encounters intense periodic cosmic storms from the galactic core, the period length is equal to the revolving period of the star around the galactic center, which normally would be of the scale of millions of years. Such intense ec loop currents around the galactic core creating $H z^{\prime}$ tilted to the galactic plane [20] as it cannot be aligned to the $L z$ states discussed earlier in section 2, that causes the stars to spiral outward from the core, would create the radiation belts at $z^{\prime}$ equatorial plane that in turn produces huge magnetic and atmospheric storms as earth revolves around the galaxy and enters, exists the radiation belt that would in turn severely affects its climatic pattern, including active volcanic eruptions inducing continental shifts during the early years of the solar system when the density of charge particles was more intense and even "ice age", when it gets out of the belt, such effects are far more intense than any changes in the contend of green house gas, such as $\mathrm{CO}_{2}$ within the planet's atmosphere. Due to this cosmic effect on our earth's climate exists, recent climate changes on earth as solely due to human created $\mathrm{CO}_{2}$ effect cannot be considered as conclusive. In fact we can make a crude model on this effect. Let us say the galactic core system orbital radius around the Milky Way core is $R$, and its revolving speed is " $W$ ". Then the approximate period $T(o)=\frac{2 \pi R}{w}$. The sun is however on the surface of the galactic doughnut tube of radius " $r$ ", and inclined to the galactic plane with angle theta.

Then the position of the sun to the galactic core is 


$$
R^{\prime}=R+r \sqrt{\sin ^{2} \theta+(1-\cos \theta)^{2}}=R+r \sqrt{2(1-\cos \theta)}
$$

There are therefore periodic effects based on the Jcurrents loops emitted from the core's curvature creation of a tilted radiation belt energetic particle hitting planets like earth as it enters and leaves the belt.

First because the solar system spirals outward from the galactic core $R$ is an increasing function of time. Thus for each orbital period, a change of the period of $D T=\frac{2 \pi}{w} \frac{\mathrm{d} R}{\mathrm{~d} t}$ occurs.

Second, the effect of the direct free charge radiation trapped in the galactic radiation belt created by the $J$ or $-J$ closed loops around the core, which must not be aligned with $L z$, the galactic angular momentum due to annihilation forbidden path overlap of the e-trino states. Hence solar activity occurs only if the galactic belt hits on the solar system, and in turn causes the solar radiation periods, which as compared to the spiral period $T(o)$ is

$$
T^{\prime}(o)=T(o)\left[\frac{r}{R}\right] \sqrt{2(1-\cos \theta)}
$$

For the Milky Way $\frac{r}{R} \gg 1$, however for the sun, $\theta$ is a small angle [20]. Thus $T^{\prime}(o)$ can be actually greater or less than the speed of the spiral of the stars in the Milky Way. Very likely such galactic influence on earth's climate change is of order of millions of year apart. Also because $R$ and $r$ actually expand with time the $J$ effect decreases with each change, hence earth's historic known major climate changes must be more severe than any that comes now, and in the future.

\section{The Boundary Effects on the $e$-Trino and Anti- $e$-Trino Pair States within a Poincare Sphere of Matter}

In the previous sections, we studied the $e$-trino, anti-e-trino opposite momentum 1'D monopole currents which are created from energy in the 5D manifold. For any totally matter enclosed sphere, the time frozen spherical boundary imposed on these pairs states would necessarily convert the open $\vec{M},-\vec{M}$ like magnetic currents into perpetual ec closed loops. The perpendicular axis $z^{\prime}$ to the closed ec loops cannot be in exact alignment to the in phase e-trino, anti-e-trino rotating perpetual pairs as we had mentioned in section 3 , that would generate a self rotation of angular momentum $L z$, as it will cause $e,-e$ annihilation due to $t>0$ only in the 5D metric. This is clearly observed in stars and planets. In fact this same restriction must also happen with the ec closed loop states around the doughnut shaped galactic core. Take the Milky Way, as example, the closed ecloops, would then generate a dipolar magnetic field $H z^{\prime}$, that is tilted to the galactic plane. Hence, all charges trapped within this $\mathrm{Hz}^{\prime}$ field created by the closed ecloops around the galactic core will give raise to a tilted radiation belt as is well known in the Milky Way to astronomers [20]. Except these trapped charges cannot spiral into the poles, unlike those charged particles in the earth's radiation belt because of the non-symmetric gravity changes for 
the doughnut geometry. It is this galactic radiation belt, that will periodically affects the periodic large climate change, such as ice age, on earth as the solar system rotates in and out of the belt around the galaxy. In the closed matter sphere, pure radial $M$ within the 3D $\times 1 \mathrm{D}$ time frozen void can no longer exist due to the reflective $M$ state leads to annihilation of the massless spinor pair. Hence if such a time frozen core retains a lot of energy, at thermal equilibrium given by Temperature $T$, and if its equivalent energy density is higher than the dense quark mass then it will continue to create mass from formation of nucleus and electrons on the inner mass shell surface, until the core $T$ is too cool to create more mass. We can treat the nuclear and electron mass formation on the inner surface of the astronomical void as if given by the magnetic monopole well in a local scale with open 5D topological representation, hence $N$ in (1.5) is given by $T$ derived from the $M$ states Bose distribution. In another word, the 5D Fermat's sum can be expressed in energy-momentum, with energy via concept of entropy further replaced by temperature. Since currents also cannot cross, due to the annihilation of the $e,-e$ pairs, hence such closed $J$ and $-J$ loops can only be on parallel and separated configuration as discussed in section 1. It is the closed Jloop feature that make $M$, the monopole resembles the individual poles of a magnetic dipole [14]. Furthermore, the dipolar magnetic field $\mathrm{Hz}^{\prime}$ generated will further requires that they be separated into north and south magnetic hemispheres due to the split of $J$ along $r$ into two ec parallel closed loops separated along $z^{\prime}$. Thus all such spherical objects will carry a dipolar $H z^{\prime}$ field. Though the $H z^{\prime}$ field strength depends on the energies carry by these ec loops is not restricted. Hence dipolar magnetic field is always found in stars and planets. Not all energy within the $3 \mathrm{D} \times 1 \mathrm{D}$ void is carried by the ecloops, we still have the pure noncharge angular momenta states carried by the $e,-e$ parallel in phase rotating pairs. Such pairs cannot be producing an angular momentum parallel to $\mathrm{Hz}^{\prime}$. In fact there is no alignment relationship. Of course, since the total $3 \mathrm{D} \times 1 \mathrm{D}$ void including the matter shell cannot have a residual angular momentum as it is created from a 5D homogeneous manifold, it means the actual matter shell must provide the exact reverse angular momentum. It is this physics that makes stars and planets having a self rotation. We have published two detail papers on the data of stars and planets [13] [14]. Astronomical bodies like our moon, appears not to have a self rotation, and a dipolar $\mathrm{H}$ field. According to the Poincare sphere model, such an object, our moon must have a very cool or very small 3D $\times 1 \mathrm{D}$ void or both. Because of that, the moon could have been initially ejected from the earth's crust with one side of its surface totally exposed to the solar wind radiation, hence has very different temperatures on the sun facing side to that opposite side. These conditions, make it unsuitable for life irrespective of its absence of an atmosphere and water. Other solar planets, except Mars are not within the life habitable solar radiation range, and therefore cannot sustain life, unless may be on their associated moons. Not all moons of planets are like ours. In fact, those objects could have a self rotation and might even sustain life, if it also receives enough photo radiation not just from the sun, but also from the 
planet it revolves around to keep part of its surface temperature at least some time above water freezing. It is always human's goal to populate outer space. But so far, our best bet is Mars. Unfortunately, Mars has a thin atmosphere, without enough oxygen and nitrogen, plus surface water, though it is not lack of water within its crust, as it's averaged surface temperature is below freezing. If our theory on the $e$-trino, anti-e-trino states in the core of planet Mars is valid, there might be possibilities for human to correct the planet's environment so as to make it suitable for the survival of life? To design the engineering on its climate modification we need to first study the physics of nuclear fusion that naturally occurs in stars and planets.

\section{The Nuclear Shell Model, Leading to Fusion and Fission}

Nuclear shell model [21] is consistent with the 5D field theory, as we discussed in section 2. Since the nucleon binding within a nucleus arises from the nucleon capture by the monopole quantum well that obeys Gauss Law, the quantum states of the nucleus must then resembles those of the atomic Bohr model. To show that we start with the projection mechanism of creating electrons and quarks, the lowest gauge confined quark states that gives us a Fermion are the proton and neutron, with its mass mainly due to the gauge constrain on the triple tensor products of the Maxwell 4 vector potentials generated by the fractional charged quark currents, known in the Gell-Mann standard model as gluon fields. Since both quarks, and the Maxwell 4 vector potentials exist only in the $4 \mathrm{D}$ space-time domain, both the proton and neutron are gauge invariant and of purely matters within the Lorentz $4 \mathrm{D}$ domain, thus they can only exist in a shell that could enclose a time frozen void of $3 \mathrm{D} \times 1 \mathrm{D}$. Their presence breaks the parity symmetry of the monopole-monopole quantum well strength. Of course the dimension of such a void has no upper and lower limits. For example, the alpha particle is like the single proton, it cannot geometrically form a closed shell, and hence do not contain a $3 \mathrm{D} \times 1 \mathrm{D}$ void. The lowest form of a nucleus with a perfect spherical void is carbon 12 [22]. In short, only large and heavy nucleus has such a void. It is therefore geometrically clear that heavy nucleus requires a very energetic $3 \mathrm{D} \times 1 \mathrm{D}$ void, and are formed from the fusion of the lighter nuclei through the Perelman-entropy mapping, and in the quantum sense the present of $V(5)$ accompanied by parity breaking of the quantum well strength. The physical reason is because $e$-trino with energy less than the threshold energy of the bare quarks masses cannot be lost instantaneous as the heavy nucleus is formed, as it can only be lost through the annihilation of $e,-e$ pairs, and emitted as photons, or as a counter example, we consider a Bethe decay of a nucleus. In this case, the neutron is converted into a proton, and an electron is emitted. Such a process changes $q$ in the nucleus, and thus $-\vec{M}$ and $-\vec{M}^{\prime \prime}$ of the quantum well, together with the Lorentz force due to $\mathrm{Hz}^{\prime}$ that breaks parity thus must further changes the $\vec{M}$ and $-\vec{M}$ parity as mentioned in ref.2. Hence nuclear Bethe decay process violates parity [23]. Unless we have such a void to house extra nucleons, and reduces the energy within the quantum well, as discussed in the previous section, such heavy nucleus cannot be created. With the same argument a super heavy nucleus would house more $e,-e$ massless 
spinors, which must be in states that give us angular momentum, and thereby the centrifugal force, that might not be possible to be contained within the void, thus leading to radiation and even breakup causing nuclear fission, as the nuclear shell binding for heavy nucleus is weakened by the proton-proton repulsive Coulomb potential as $\frac{N !}{2}$, where $N$ is the atomic number of the nucleus. Leading to instability in very heavy elements, such as Uranium. In conclusion, fission releases energy from the nucleus, while fusion reduces the overall energy of the nucleus and stabilized the void containing energy. In fact this phenomena carries over to astronomical objects, such as the inner shell surface of stars and planets. Thus if we wants to increase the planet's core energy, we may employ a controlled triggered nuclear fission process, that does not activates the outward thermal pressure significantly unevenly through volcanic eruptions and blow up too much of the planet's crest, yet raising the core temperature hot enough to trigger the nuclear fusion by forming from the nuclear residual fragments from fission into more stable non radiative heavy elements, such as iron and noble metals. In so doing we not only can activate the $e$-trino, anti-e-trino in phase angular momentum value, but also a change in the planet's moment of inertia from the shell mass density change, which is accompanied usually also by chemically changing the surface elements and in its atmosphere. In turn would even allow for increase in heat retaining $\mathrm{CO}_{2}$ green house gas, plus changes in oxygen and nitrogen composition due to decomposition of oxides and nitrates from the crest. And with water vapor if the surface is above freezing, after cooling creates surface water pools, forming seas and lakes and streams. To achieve a man make planet core temperature change via nuclear fission and fusion for example on Mars, [see recent news article on Mars.] require solving many technical problems. For example, how to send a carefully controlled nuclear fission device into the planet's core intact and then trigger it. If we can achieve and solve these technical problems, then perhaps, it is the best humanitarian reward we earned from our previous technological development on creating nuclear weapons?

\section{Conclusion-The Mathematical Theorems}

It is best to summarize the important mathematical theorems that are employed in the 5D theory we present in this paper. First and foremost, all homogeneous manifolds satisfy the Fermat's Last Theorem. So does the 5D. Second, all space and time measurements must obey the uncertainty principle, thus the Fermat's sum gives the same dimensional homogeneous quadratic operator, with only plane wave solutions. The boundary on such a homogeneous space-time manifold is a manifold that is one space dimension lower. Thus for the $5 \mathrm{D}$ homogeneous space-time manifold, it is the $4 \mathrm{D}$ homogeneous Maxwell manifold. It is this theorem that provides us with the 5th component vector potential, the magnetic monopoles. The Perelman-Ricci Flow Theorem allows for the connecting of 4D Maxwell domains into a Lorentz 4D doughnut domain, thus chopping the single $5 \mathrm{D}$ universe into interconnected $t \mathrm{U} 3 \mathrm{D} \times 1 \mathrm{D}$ space-time topology with the initial absolute zero magnitude point of the Fermat's sum into a line of zero dimension, similarly, with the energy-momentum metric, the initial 
single infinite energy point from the original 5D into disconnected multiple zero spread points of infinite energy. These mappings are mathematically profound, because only 0 and $\infty$ can be divided arbitrarily into, no matter how many and remain exactly 0 and infinite. Lastly, if in each $5 \mathrm{D}$ manifold, $t$ is fixed, then it follows the $4 \mathrm{D}$ space that is arbitrarily divided and separated into Poincare spheres with time independent voids of $3 \mathrm{D} \times 1 \mathrm{D}$. Such topological subspaces are obtained via the Perelman-entropy mapping. Such mapping must follow from the closing up of the connected doughnut $4 \mathrm{D}$ manifolds. However, none of these mathematical theorems can give us the space to time and space to space dimension reduction projections, as projection transformation has no inverse, and must be enacted, meaning that it can only happen by command, an implication similar to the creation of the 5D metric itself, coming from the universe creator.

\section{Acknowledgements}

We thank Ms. Winnie So, Ms. Elize Yeung and Dr. Anthony Cheng for their help in typing this manuscript.

\section{Conflicts of Interest}

The authors declare no conflicts of interest regarding the publication of this paper.

\section{References}

[1] Wong, K.W., Dreschhoff, G.A.M. and Jungner, H. (2014) The Five Dimension Space-Time Universe-A Creation and Grand Unified Field Theory Model. Scientific Research Publishing, USA.

[2] Gell-Mann, M. (1964) Physical Review Letters, 12, 155. https://doi.org/10.1103/PhysRevLett.12.155

[3] Wong, K.W., Dreschhoff, G.A.M. and Jungner, H.J.N. (2012) The J/I Meson and the Missing Heavy Baryon Octet. ArXiv:1204.0238v1.

[4] Wong, K.W., Dreschhoff, G. and Jungner, H. (2012) Journal of Modern Physics, 3, 1450-1457. https://doi.org/10.4236/jmp.2012.310179

[5] Wong, K.W., Dreschhoff, G. and Jungner H. (2015) Journal of Modern Physics, 6, 890-901. https://doi.org/10.4236/jmp.2015.67093

[6] Perelmann, G. (2002) The Entropy Formula for the Ricci Flow and Its Geometric Applications. arXiv:math.DG/0211159 [math.DG]

[7] Perelmann, G. (2003) Ricci Flow with Surgery on Three-Manifolds. arXiv:math.DG/0303109 2003a [math.DG]

[8] Diklan, J. (2019) You Are Disturbing Me-I'm Picking Mushrooms! Academia.edu. Jan. 2019.

[9] Aczel, A.D. (1997) Fermat's Last Theorem: Unlocking the Secret of an Ancient Mathematical Problem. Penguin, London, $147 \mathrm{p}$.

[10] Maxwell, J.C. (1864) A Dynamical Theory of the Electromagnetic Field. The Royal Society Transactions, Volume CLV. Printed in: The Scientific Papers of James Clerk Maxwell (W.D. Niven), Two Volumes Bound as One, Volume One, New York, Dover Publications, Inc., 1890, 526. Also refer to J. C. Maxwell (1865) Philosophical 
Transactions of the Royal Society of London, 155, 459-512.

[11] Wong, K.W., Dreschhoff, G., Jungner, H., Fung, P.C.W. and Chow, W.-K. (2018) Physics Essays, 31, 493-495. https://doi.org/10.4006/0836-1398-31.4.493

[12] Higgs, P.W. (1964) Physical Review Letters, 13, 508. https://doi.org/10.1103/PhysRevLett.13.508

[13] Fung, P.C.W. and Wong, K.W. (2015) Journal of Modern Physics, 6, 2303-2341. https://doi.org/10.4236/jmp.2015.615235

[14] Fung, P.C.W. and Wong, K.W. (2017) Journal of Modern Physics, 8, 668-746. https://doi.org/10.4236/jmp.2017.84045

[15] Wheeler, J.A. (1955) Physical Review, 97, 511-536. https://doi.org/10.1103/PhysRev.97.511

[16] Goldstein, A. et al. (2017) The Astrophysical Journal Letters, 848, L14. https://doi.org/10.3847/2041-8213/aa8f41

[17] Chernyakova, M. (2019) Nature, 567, 318-320.

[18] Yang, X.L., Guo, S.H., Chan, F.T., Wong, K.W. and Ching, W.Y. (1991) Physical Review A, 43, 1186-1196. https://doi.org/10.1103/PhysRevA.43.1186

[19] Gou, S.H., Yang, X.L., Chan, F.T., Wong, K.W. and Ching, W.Y. (1991) Physical Review A, 43, 1197-1205. https://doi.org/10.1103/PhysRevA.43.1197

[20] Chen, X.D., et al. (2018) Nature Astronomy, 10, 1038.

[21] Goldhammer, P. (1963) Reviews of Modern Physics, 35, 40-107. https://doi.org/10.1103/RevModPhys.35.40

[22] Wong, K.W. (1964) Journal of Mathematical Physics, 6, 537.

[23] Lee, T.D. and Yang, C.N. (1957) Physical Review, 105, 1671-1675. 